\title{
Editorial: Data-Driven Cognitive Manufacturing-Applications in Predictive Maintenance and Zero Defect Manufacturing
}

\author{
Dimitris Kiritsis ${ }^{1 *}$, Oscar Lazaro ${ }^{2}$, Melinda Hodkiewicz ${ }^{3}$, Jay Lee ${ }^{4}$ and Jun $\mathrm{Ni}^{5}$ \\ ${ }^{1}$ École Polytechnique Fédérale de Lausanne, Lausanne, Switzerland, ${ }^{2}$ Innovalia Association, Bizkaia, Spain, ${ }^{3}$ University of \\ Western Australia, Perth, WA, Australia, ${ }^{4}$ University of Cincinnati, Cincinnati, $\mathrm{OH}$, United States, ${ }^{5}$ University of Michigan, Ann \\ Arbor, MI, United States
}

Keywords: big data for factories, closed loop lifecycle management, zero defect manufacturing, predictive maintenance, semantic technologies, industrial ontologies, industrial Al, artificial intelligence

Editorial on the Research Topic

Data-Driven Cognitive Manufacturing - Applications in Predictive Maintenance and Zero Defect Manufacturing

Closed-Loop Lifecycle Management (CL2M) is an integral part of the circular economy. Managing the CL2M enables manufacturers and associated digital factories to connect in-service issues back to process conditions and product information at manufacturing and other stages of the life cycle with the aim of having Zero Defect Manufacturing (ZDM).

ZDM can be implemented through two approaches: product-oriented and process-oriented ZDM. Product-oriented ZDM studies defects in the actual parts., Process-oriented ZDM studies defects in the manufacturing equipment that have led, or might lead to product defects this is implemented through Predictive Maintenance.

The Industrial Internet of Things (IIoT) and associated computing continuum Cloud and Edge Technologies and Industrial AI (Artificial Intelligence) provide valuable data for Predictive Maintenance and product-oriented ZDM. Associated to that, ontologies and associated semantic technologies such as Knowledge Graphs are rapidly becoming popular in various domains and applications to deal with adding semantic meaning to this data and enable reasoning and queries.

All of the above is making the smart maintenance and manufacturing development with increasing "cognitive" and "predictive" characteristics to augment the human-machine collaboration.

In this Research Topic, we present a compilation of eight papers presenting and demonstrating results of recent research and innovation activity in a variety of topics within Data Driven Cognitive Manufacturing with applications in ZDM and Predictive Maintenance.

The first paper, "Product Quality Improvement Policies in Industry 4.0: Characteristics, Enabling Factors, Barriers, and Evolution Toward Zero Defect Manufacturing" by Foivos Psarommatis, Sylvain Prouvost, Gökan May and Dimitris Kiritsis presents a literature review on the implementation of these philosophies to improve quality of processes and products in a system, and also covers the commonalities and differences with Zero Defect Manufacturing (ZDM) philosophy. In this study, 45 articles have been analyzed. A categorization of quality improvement methods and the way toward ZDM is also presented and discussed.

The second paper, "Physically Inspired Data Compression and Management for Industrial Data Analytics" by Ramin Sabbagh, Zicheng Cai, Alec Stothert and Dragan Djurdjanovic describe a novel method that facilitates automated signal parsing into a set of exhaustive and mutually exclusive 
segments, which is coupled with extraction of physically interpretable signatures that characterize those segments. The resulting numerical signatures can be used to approximate a wide range of signals within some arbitrary accuracy, thus effectively turning the aforementioned signal parsing and signature extraction procedure into a signal compression process. Application to multiple large datasets of sensor readings collected from several advanced manufacturing plants showed the feasibility of physics-inspired compression of industrial data.

The aim of the third paper, "Ontology-Based Context Modeling in Physical Asset Integrity Management" by Ali AlShdifat, Christos Emmanouilidis, Muhammad Khan and Andrew G. Starr is 2-fold: to analyse current approaches to addressing IoT context information management, mapping how context-aware computing addresses key challenges and supports the delivery of monitoring solutions; and to develop a maintenance context ontology focused on failure analysis of mechanical components so as to drive monitoring services adaptation. The approach is demonstrated by applying the ontology on an industrially relevant physical gearbox test rig.

The fourth paper, "Predictive Maintenance for Injection Molding Machines Enabled by Cognitive Analytics for Industry 4.0 " by Vaia Rousopoulou, Alexandros Nizamis, Thanasis Vafeiadis, Dimosthenis Ioannidis and Dimitrios Tzovaras introduces a cognitive analytics, self- and autonomous-learned system bearing predictive maintenance solutions for Industry 4.0. A complete methodology for realtime anomaly detection on industrial data and its application on injection molding machines are presented.

The fifth paper, "Prognostics and Health Management of Industrial Assets: Current Progress and Road Ahead" by Luca Biggio and Iason Kastanis presents a thorough review of existing works both in the contexts of fault diagnosis and fault prognosis, highlighting the benefits and the drawbacks introduced by the adoption of AI techniques. The goal of the authors in this paper is to highlight potentially fruitful research directions along with characterizing the main challenges that need to be addressed in order to realize the promises of AI-based Prognostics and Health Management systems.

In the sixth paper, "Implementation and Transfer of Predictive Analytics for Smart Maintenance: A Case Study" by Sebastian Von Enzberg, Thanasis Naskos, Ifigeneia Metaxa, Daniel Köchling and Arno Kühn, the authors present a case study motivated by a typical maintenance activity in an industrial plant. The paper focuses on the crucial aspects of each phase of the Predictive Maintenance implementation process, towards the holistic integration of the solution within a company. A concept is derived for the model transfer to a different factory. This is illustrated by practical examples from a lighthouse factory within the BOOST $4.0 \mathrm{H} 2020$ project.

The seventh paper, "Intelligent Predictive Maintenance and Remote Monitoring Framework for Industrial Equipment based on Mixed Reality" by Dimitris Mourtzis, John Angelopoulos and Nikos Panopoulos propose an approach for the modelling, design and development of a Predictive Maintenance and Remote Monitoring system, based on the utilization of AI algorithms for the data acquisition, fusion, and postprocessing. In addition to that, the proposed framework will integrate a Mixed Reality application for the intuitive visualization of the data, that will ultimately facilitate production and maintenance engineers to monitor the condition of the machines and most importantly to get an accurate prediction of the oncoming failures.

The final paper, "RECLAIM: Towards new era of refurbishment and re-manufacturing of industrial equipment" by Angeliki Zacharaki, Thanasis Vafeiadis, Nikolaos Kolokas, Yuchun Xu, Michael Pesch, Dimosthenis Ioannidis and Dimitrios Tzovaras presents a new idea on refurbishment and remanufacturing based on big data analytics, machine learning, predictive analytics and optimization models using deep learning techniques and digital twin models with the aim of facilitating the stakeholders to make informed decisions about whether to remanufacture, upgrade or repair heavy machinery that is towards its end-of-life.

\section{AUTHOR CONTRIBUTIONS}

All authors listed have made a substantial, direct and intellectual contribution to the work, and approved it for publication.

Conflict of Interest: The authors declare that the research was conducted in the absence of any commercial or financial relationships that could be construed as a potential conflict of interest.

Copyright (c) 2021 Kiritsis, Lazaro, Hodkiewicz, Lee and Ni. This is an open-access article distributed under the terms of the Creative Commons Attribution License (CC $B Y$ ). The use, distribution or reproduction in other forums is permitted, provided the original author(s) and the copyright owner(s) are credited and that the original publication in this journal is cited, in accordance with accepted academic practice. No use, distribution or reproduction is permitted which does not comply with these terms. 